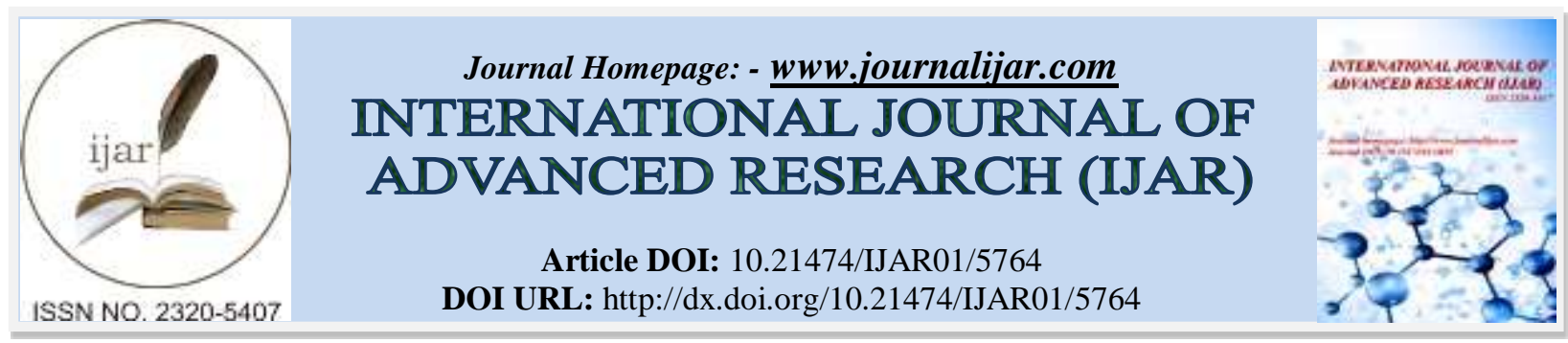

RESEARCH ARTICLE

\title{
THE DYNAMICS OF THE ROTARY MANUALLY MOTORIZED MOWER WITH THE ROTARY WORK ORGAN.
}

\author{
Zaur Fhutkaradze ${ }^{1}$, Merab Mamuladze ${ }^{2}$, Soso Tavberidze ${ }^{3}$ and Rezo Vasadze ${ }^{4}$. \\ 1. Associate Professor, Batumi State Maritime Academy. \\ 2. Associate Professor, Batumi Shota Rustaveli State University. \\ 3. Associate Professor, Tsereteli State University. \\ 4. Professor, Tbel Abuseridze State University.
}

\section{Manuscript Info}

Manuscript History

Received: 04 September 2017

Final Accepted: 06 October 2017

Published: November 2017

Key words:-

Engine; Dynamic processes; Moment of inertia; Rotary work organ; Vibrating strokes.

\section{Abstract}

Dynamics of the main factors working on the internal combustion engine, during rotary manually-motorized mower's working time, on which the normal providence of the working technological process of the mower is dependent, namely, factors of the dynamic processes influence the following: during the working process of the mower, extra vibrating strokes are excluded; during the working process risks of appearance of internal issues of the vehicle are eliminated and working of operator is convenient.

Copy Right, IJAR, 2017,. All rights reserved.

\section{Introduction:-}

an approach is developed for sensitivity analysis of inertial parameters. It is based on the reformed equations of motion linear in terms of the inertial parameters, which are transformed to dimensionless inertial parameters. Based on an eigenvalue analysis, directions of the parameter space are found along which maximum/minimum changes are achieved on the generalized forces, i.e., on the dynamics of the system. Directional derivatives of the generalized force vector in these directions characterize how each inertial parameter contributes to the system dynamics. (Saeed Ebrahim, Arash Haghi 2013). Accurate automatic guidance of towed implements is important for performing agricultural fieldoperations and for gaining the ultimate benefit from such systems. The study of open and closed loop system responses of a vehicle-implement system can be helpful in the design of practical guidance controllers. Open loop analys is of the kinematic and dynamic model srevealed that the high reorder dynamics captured by the tractor and implement dynamic model had an impact on simulated responses at higher operating velocities and on higher input frequencies. In addition, a dynamic model with tire relaxation length dynamics was also studied. The various model responses were compared with the experimental responses. Closed loop system characteristics were studied by using a linear quadratic regulator (LQR) controller. The tractor position and heading and implement the ading states along with respective ratestates were fed back toclose the loop. Steering dynamics were also added to the dynamic model closed loop analysis, which helped to achieve a realistic closed loop steering angle history. The closed loop system dynamics became faster as the forward velocity was increased. The open and closed loop response analysis performed in this work provided an understanding about the system at various forward velocities, which will help to design and develop efficient and robust tractor and towed implement guidance controller (Feng, L., Y. He Y. Bao, and H. Fang (2005). Tractor and towed implement system models have become increasingly important for model-based guidance controller design, virtual prototyping, and operator-and-hardwarein-loop simulation. Various tractor and towed implement models have been proposed in the literature which contain uncertain or time-varying parameters. Sensitivity analysis was used to identify the effect of system parameter 
variation on system responses and to identify the most critical system parameters. Sensitivity analysis was performed with respect to three tire cornering stiffness parameters, three tire relaxation length parameters, and two implement inertial parameters. Overall, the system was most sensitive to the tire cornering stiffness parameters and least sensitive to the implement inertial parameters. In general, the variation in the input parameters and the system state variables were related in a non-linear fashion. With the nominal parameter values for a MFWD tractor, a single axle grain cart, and corn stubble surface conditions, a $10 \%$ variation in cornering stiffness parameters caused a $5 \%$ average variation in the system responses whereas an $80 \%$ change in cornering stiffness parameters caused an $80 \%$ average variation at $4.5 \mathrm{~m} / \mathrm{s}$ forward velocity. If a $10 \%$ average variation in system responses is acceptable, the cornering stiffness parameters and implement inertial parameters must be estimated within $20 \%$ and $30 \%$ of actual/nominal values respectively. The relaxation length parameters have to be within $75 \%$ of the nominal values (Kim, Y. C., K. H. Yun, K. D. Min; Y. S. Byun; and J. K. Mok (2007). development process of virtual experiment was analyzed in this paper. Then a virtual experiment system for agricultural equipment was founded by using Multi Gen Creator, Vega Prime software and VC++ programming language. The system running process was introduced, together with tractor 3D modeling, operation data acquisition, dynamic modeling and drive, and collision detection. Furthermore, visual drive, synchronous display, tractor dynamics solution in network and other key techniques related to virtual experiment were discussed as well. The system provides a new method to tractor performance experiment, and the experimental results show stable operation of the system with the reliability and validity(Zang Yu ; Zhu Zhongxiang ; Song Zhenghe ; Xu Jing ; Mao Enrong 2010).

\section{Materials and Methods:-}

The perspectives of development of agrarian processes in Georgia showed that cattle breeding is one of the priority areas in agriculture, which leads to production, processing and taking livestock products to the customer markets. Therefore, in a small farming and peasant households, to supply the animals with food supply in a timely, efficient and economical manner, remains the priority. This is directly dependent on the farmer's economic profit, in areas where there are hillsides the wide area mowers are not efficient to operate. It is recommended to use mowers with lower capacity to conduct mowing, which will solve the above problems.

The purpose of the research of the scientific thesis is to calculate the basic parameters of internal dynamic processes of the mowers with small internal combustion engine, for using them in mountainous conditions.

During the operation of the rotary manually motorized mower, attention should be paid to the main factors, such as the moment of inertia, the moment of setting driver in motion, the angular velocity of the driver and the coefficient of the unevenness. These characteristics increase the quality of technological processes and reduce unwanted extra vibrating strokes in the vehicle.

For technical facilities of small-scale mechanizations with one working body, a two-axle equivalent design scheme is used to drive the motor or the flexible shaft (Fig. 1)

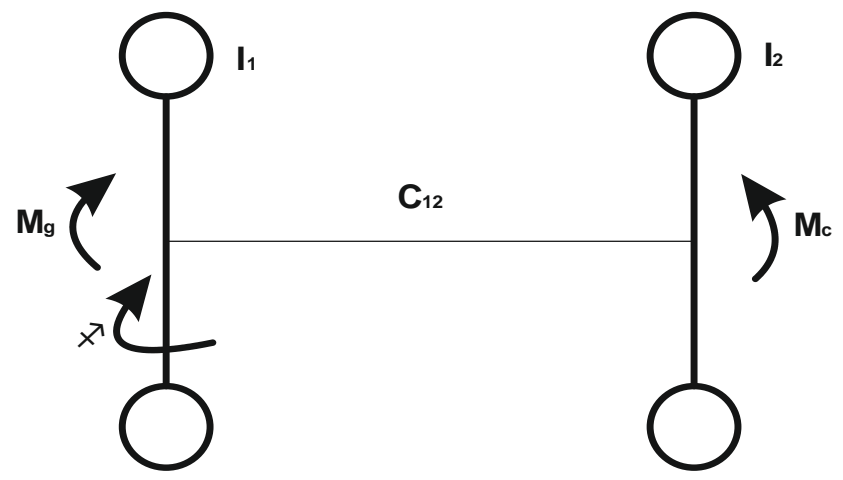



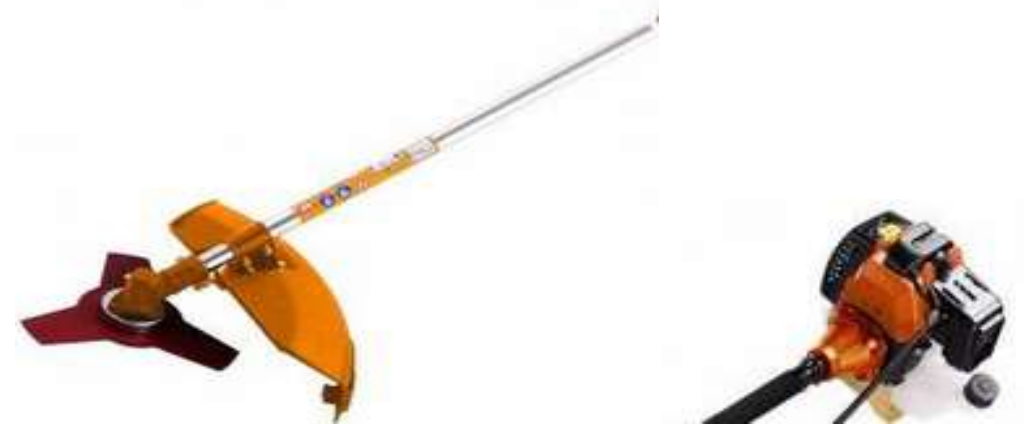

The moment of inertia in low motorized manual technical means is constant and researches state (Pankratov S. A. 1976) that the moment of inertia is functionally expressed as follows:

$$
\begin{aligned}
f(\varphi)=\frac{\frac{1}{2} \cdot \frac{d \mathfrak{J}(\varphi)}{\mathfrak{J}(\varphi)}+B}{\mathfrak{J}(\varphi)} & \\
q(\varphi) & =\frac{M_{C}(\varphi)-A}{\mathfrak{J}(\varphi)} .
\end{aligned}
$$

Hence, in low motorized manual technical means we will have:

$$
\begin{aligned}
\mathcal{f}(\varphi) & =\frac{B}{\mathfrak{J}} \\
q(\varphi) & =\frac{M_{C}(\varphi)-A}{\mathfrak{J}}
\end{aligned}
$$

Where $\mathrm{I}$ is moment of inertia, $\mathrm{M}_{\mathrm{C}}$ - the moment of setting driver in motion, and $\mathrm{A}$ and $\mathrm{B}$ are coefficients, which are calculated according to the quality of the slider mechanism.

Let us consider the case when the moment of resistance is permanent $M_{C}(\varphi)=M_{C}=$ const . In initial conditions $t=0, \omega=\omega_{0}$. Then, the angular velocity is determined according to the following formula (Pankratov S. A. 1976):

$$
\omega(\varphi)=\sqrt{\exp \left[-2 \int_{0}^{\varphi} f(\varphi) d(\varphi)\right]\left\{c-2 \int_{0}^{\varphi} q(\varphi) \exp \left[2 \int_{0}^{\varphi} f(\varphi) d \varphi\right] d \varphi\right\}}
$$

For our case it will be:

$\omega(\varphi)=\sqrt{\exp \left[-\frac{2 B \varphi}{\mathcal{J}}\right]\left\{\omega_{0}-\frac{2\left(M_{C}-A\right)}{\mathcal{J}} \int_{0}^{\varphi} \exp \left[\frac{2 B \varphi}{\mathfrak{J}}\right] d \varphi\right\}}$

From where:

$$
\begin{aligned}
\omega(\varphi) & =\sqrt{\exp \left[-\frac{2 B \varphi}{\mathfrak{J}}\right]\left\{\omega_{0}^{2}-\frac{M_{C}-A}{B}\left[\exp \left(\frac{2 B \varphi}{\mathfrak{J}}\right)-1\right]\right\}} \\
\text { Or } \omega(\varphi) & =\sqrt{\omega_{0}^{2} \exp \left[-\frac{2 B \varphi}{\mathfrak{J}}\right]-\frac{M_{C}-A}{B}\left[1-\exp \left(-\frac{2 B \varphi}{\mathfrak{J}}\right)\right]}
\end{aligned}
$$


Theoretically starting movement will be received when:

$\varphi \rightarrow \infty$, for this, the angular velocity in the beginning of the movement is calculated with the following equation:

$$
\omega=\sqrt{\frac{A-M_{C}}{B}}
$$

The moment of resistance $M_{C}(\varphi)$ is expressed with the following formula [2]:

$$
M_{C}(\varphi)=\frac{\alpha}{2}+\sum_{n=1}^{m}\left(\alpha_{n} \cos \varphi+b_{n} \sin n \varphi\right)
$$

Where, $\alpha_{0}, \alpha_{n}, b_{n}(n=1,2, \ldots m)$ are the constant values and are calculated with the following formulas:

$$
\left.\begin{array}{l}
\alpha_{0}=\frac{1}{T} \int_{0}^{T} M_{C}(\varphi) d \varphi \\
\alpha_{n}=\frac{2}{T} \int_{0}^{T} M_{C}(\varphi) \cos n \varphi d \varphi \\
b_{n}=\frac{2}{T} \int_{0}^{T} M_{C}(\varphi) \sin n \varphi d \varphi
\end{array}\right\}
$$

Where - T is period $\left(T=\frac{2 \pi}{\omega}\right)$. Then, (4) the formula will be displayed as follows:

$$
q(\omega)=\frac{1}{\mathfrak{J}}\left[\frac{\alpha_{0}}{2}-A+\sum_{n=1}^{m}\left(\alpha_{n} \cos n \varphi-b n \sin n \varphi\right)\right]
$$

For initial conditions based on common decisions $t=0, \omega=\omega_{0}$ then,

(13)

$$
\omega(\varphi)=\sqrt{\exp \left[-\frac{2 B \varphi}{\mathfrak{J}}\right]\left\{\omega_{0}^{2}-\frac{2}{\mathfrak{J}} \int_{0}^{\varphi}\left[\frac{\alpha_{0}}{2}-A+\sum_{n=1}^{m}\left(\alpha_{n} \cos n \varphi-b n \sin n \varphi\right)\right] \exp \left(\frac{2 B \varphi}{\mathfrak{J}}\right) d \varphi\right\}}
$$

If we convert (13), the formula will receive the formula of the angular velocity:

$$
\begin{aligned}
\omega(\varphi)= & \sqrt{\left[\omega_{0}^{2}+\frac{\alpha_{0}-2 A}{2 B}+\frac{2}{\mathcal{J}} \sum_{n=1}^{m} \frac{\frac{2 \alpha_{n} B}{\mathcal{J}}-b_{n} n}{\left(\frac{2 B}{\mathcal{J}}\right)^{2}+n^{2}}\right] \exp \left(-\frac{2 B \varphi}{\mathcal{J}}\right)-\frac{\alpha_{0}-2 A}{2 B}-} \\
& -\frac{2}{\mathfrak{J}} \sum_{n=1}^{m} \frac{\left(\frac{2 \alpha_{n} B}{\mathfrak{J}}-b_{n} n\right) \cos \varphi+\left(\frac{2 b_{n} B}{\mathfrak{J}}+\alpha_{n} n\right) \sin \varphi}{\left(\frac{2 B}{\mathfrak{J}}\right)^{2}+n^{2}}
\end{aligned}
$$

When starting the movement $\lim _{\varphi \rightarrow \infty} \exp \left(-\frac{2 B \varphi}{\mathfrak{T}}\right)=0$

Therefore: 


$$
\omega(\varphi)=\sqrt{\frac{2 A-\alpha_{0}}{2 B}-\frac{2}{\mathfrak{J}} \sum_{n=1}^{m} \frac{\left(\frac{2 \alpha_{n} b}{\mathfrak{J}}-b_{n} n\right) \cos n \varphi+\left(\frac{2 b_{n} B}{\mathfrak{J}}+\alpha_{n} n\right) \sin n \varphi}{\left(\frac{2 B}{\mathfrak{J}}\right)^{2}+n^{2}}}
$$

The angular acceleration equation (Gernet M. M. and others 1972) is equal to:

$$
\varepsilon(\varphi)=\left\{\boldsymbol{f}(\varphi) \exp \left[-2 \int_{0}^{\varphi} \mathcal{f}(\varphi) d \varphi\right] \cdot\left[C-2 \int_{0}^{\varphi} q(\varphi) \exp \left(2 \int_{0}^{\varphi} f(\varphi) d \varphi\right)\right]+q(\varphi)\right\}
$$

The value of angular acceleration of the mower at the period of transition will be the following:

$$
\begin{gathered}
\varepsilon(\varphi)=-\frac{B}{\mathfrak{J}}\left\{\left[\omega_{0}^{2}+\frac{\alpha_{0}-2 A}{2 B}+\frac{2}{\mathfrak{J}} \sum_{n=1}^{m} \frac{\frac{2 \alpha_{n} B}{\mathfrak{J}}-b_{n} n}{\left(\frac{2 B}{\mathfrak{I}}\right)^{2}+n^{2}}\right] \exp \left(-\frac{2 B \varphi}{\mathfrak{J}}\right)-\frac{\alpha_{0}-2 A}{2 B}-\right. \\
\left.-\frac{2}{\mathfrak{J}} \sum_{n=1}^{m} \frac{\left.\left(\frac{2 \alpha_{n} b}{\mathfrak{J}}-b_{n}\right) \cos n \varphi+\left(\frac{2 b_{n} B}{\mathfrak{J}}+\alpha_{n} n\right) \sin n \varphi\right)}{\left(\frac{2 B}{\mathfrak{J}}\right)^{2}+n^{2}}\right\}-\frac{1}{\mathfrak{J}}\left[\frac{\alpha_{0}}{2}-A+\sum_{n=1}^{m}\left(\alpha_{n} \cos \varphi+b_{n} \sin n \varphi\right)\right] .
\end{gathered}
$$

(17)

For determination of the experimental angular velocity (17) formula can be considered in reverse:

$$
\left.\begin{array}{l}
\frac{2 \alpha_{n} B}{\mathfrak{J}}-b_{n} n=\mathcal{Z}_{n} \sin \alpha_{n} \\
\frac{2 b_{n} B}{\mathfrak{J}}+\alpha_{n} n=\mathcal{Z}_{n} \cos \alpha_{n}
\end{array}\right\}
$$

Where:

$$
\begin{gathered}
z_{n}=\frac{\sqrt{\left(\alpha_{n}^{2}+b_{n}^{2}\right)\left(4 B^{2}+n^{2} \mathcal{J}^{2}\right)}}{\mathfrak{J}} \\
\operatorname{tg} \alpha_{n}=\frac{2 \alpha_{n} B-b_{n} n \mathcal{J}}{2 B_{n} B+b_{n} n \mathcal{J}}
\end{gathered}
$$

Therefore, (15) the formula will be displayed as follows:

$$
\omega(\varphi)=\sqrt{\frac{2 A-\alpha_{0}}{2 B}-2 \sum_{n=1}^{m} \sqrt{\frac{\alpha_{n}^{2}+b_{n}^{2}}{4 B^{2}+n^{2} \mathcal{J}^{2}}}} \cdot \sin \left(n \varphi+\alpha_{n}\right)
$$

This formula shows that the angular velocity of the drive is maximum, when the phase angle is $n \varphi+\alpha_{n}=\frac{3 \pi}{2}$ and minimum, when $-n \varphi+\alpha_{n}=\frac{\pi}{2}$, and experimental values are: 


$$
\begin{aligned}
& \omega_{\text {min }}=\sqrt{\frac{2 A-\alpha_{0}}{B}-2 \sum_{n=1}^{m} \sqrt{\frac{\alpha_{n}^{2}+b_{n}^{2}}{4 B^{2}+n^{2} \mathfrak{J}^{2}}}} \\
& \omega_{\text {max }}=\sqrt{\frac{2 A-\alpha_{0}}{B}+2 \sum_{n=1}^{m} \sqrt{\frac{\alpha_{n}^{2}+b_{n}^{2}}{4 B^{2}+n^{2} \mathfrak{J}^{2}}}}
\end{aligned}
$$

In order to prevent technological processes and vibrations, in the manually motorized mowers it is necessary to catch the speed of the drive arbor within the norms and the permissible range of variation of the angular velocity is characterized by the unevenness coefficient (Artobolevsky I. I. 1975)

$$
\delta=\frac{2\left(\omega_{\max }-\omega_{\min }\right)}{\omega_{\max }-\omega_{\min }}
$$

Based on calculations:

$$
\begin{aligned}
& \alpha_{1}=\frac{1}{T} \int_{0}^{T} M_{C}(\varphi) d \varphi \\
& b_{1}=\frac{2}{T} \int_{0}^{T} M_{C}(\varphi) d \varphi \\
& \mathfrak{J}=\int_{0}^{m} \rho^{2} d m
\end{aligned}
$$

Considering that the number of rotations of the cranked arbor of the engine (ТАИГА-214Э) is 6500-7000 rotations $/ \mathrm{min}$. then, $M_{C}=0,8 \mathrm{~kg} . \mathrm{m}^{2}, \mathfrak{J}=0,25 \mathrm{~kg} . \mathrm{m}^{2}, M_{n}=0,78 \mathrm{~kg} . \mathrm{m}^{2}$.

Then, $A=\frac{0,8 \cdot 680-0,78 \cdot 730}{680-730}=0,4$

$$
\begin{aligned}
& B=0,0000014 \\
& \omega_{\text {min }}=\sqrt{\frac{2 \cdot 0,4-0,06}{0,0000014}-2 \sqrt{\frac{0,06^{2}+0,08^{2}}{4 \cdot 0,0000014^{2}+1^{2} \cdot 0,25^{2}}}}=\sqrt{\frac{0,7}{0,0000014}-2 \sqrt{\frac{0,009}{0,06}}}=706,1 \quad\left(\mathrm{~s}^{-1}\right) \\
& \delta=\frac{2(707,1-706,1)}{707,1-706,1}=2
\end{aligned}
$$

\section{Summary:-}

In the scientific paper the moment of inertia of the mower's drive, the moment of setting driver in motion, the minimum and maximum angular velocities when drive is loaded and unevenness coefficient during the work period is calculated. All of these represent a basis for eliminating extra vibrations, risks of appearance of the internal issues of the vehicle and providing more convenient work of the operator, during mowing process when choosing inelastic arbors. 


\section{References:-}

1. Saeed Ebrahim, Arash Haghi- Characterization of the contribution of inertial parameters to the dynamics of multibody systems. Multibody System Dynamics, December 2013, Volume 30, Issue 4, pp 449-460.

2. Feng, L., Y. He Y. Bao, and H. Fang - Development of trajectory model for a tractor-implement system for automated navigation applicati ons. Instrumentation and Measurement Technology Conference, Ottawa, Canada, May 17-19.

3. Kim, Y. C., K. H. Yun, K. D. Min; Y. S. Byun; and J. K. Mok - A lateral dynamic model of an all wheel steering bimodal vehicle. International Conference on Control, Automation and Systems, Seoul, Korea, Oct. 1720.

4. Zang Yu ; Zhu Zhongxiang ; Song Zhenghe ; Xu Jing ; Mao Enrong- Study on virtual experiment methods of agricultural equipment based on Vega Prime. International Conference on Advanced Technology of Design and Manufacture (ATDM 2010) Nov. 2010.

5. Pankratov S. A. - Dynamics of machines for open mining and excavation works - M. "Mechanical Engineering". 1976 year.

6. Gernet M. M. and others - Determination of moments of inertia. M. "Mechanical Engineering". 1976 year.

7. Artobolevsky I. I. - Theory of mechanisms and machines. M. "Mechanical Engineering". 1975 year.

8. Ustinov A. N. - Agricultural technology. M. 2008 year. 